\title{
La motivación como una percepción de calidad en dos asignaturas de investigación educativa de la UNED, Costa Rica
}

Warner Ruiz Chaves

Cátedra de Investigación Educativa, Universidad Estatal a Distancia (UNED); warner.ruiz@gmail.com

Recibido: 11 de junio del 2015 Corregido: 06 de agosto del 2015

Aceptado: 16 de setiembre del 2015

\begin{abstract}
Resumen
Las asignaturas impartidas mediante entornos virtuales de aprendizaje posibilitan el desarrollo de muchas opciones de formación a los sistemas educativos modernos. Ante tal panorama, es importante conocer y estudiar los diferentes elementos que pueden propiciar ofertas formativas de calidad, no solo para el centro educativo mismo, sino desde la perspectiva de quien cursa dichas ofertas ya que el solo hecho de realizar un curso en línea con apoyo de recursos informáticos no garantiza el éxito. A este respecto, el presente documento realiza una breve descripción de la percepción de calidad que poseen los estudiantes de las asignaturas de "Investigación Educativa" y "Administración del Currículo" de la Universidad Estatal a Distancia de Costa Rica (UNED) sobre la motivación, elemento necesario para el aseguramiento de la calidad en todo proceso formativo no presencial. La investigación se llevo a cabo durante los años académicos 2012 y 2013.
\end{abstract}

Palabras Claves: Motivación, Internet, Educación, Curso en línea, Educación Virtual, UNED, Calidad.

\section{A MANERA DE INTRODUCCIÓN}

La educación universitaria a distancia, con el apoyo de entornos virtuales ha ido evolucionando rápidamente en los últimos años en muchos centros de estudios superiores. La facilidad por medio de la cual se accede a un entorno sin necesidad de trasladarse físicamente

\section{Abstract \\ Motivation as an enhancer of perception of the quality of two virtual courses on educational research at the UNED, Costa Rica}

The courses through virtual learning environments enable the development of many training options of modern education systems. Under such conditions it is important to know and study the different elements that can promote quality training programs in education, not only for the learning centers, but from the perspective of who is in such training. On that matter, merely taking an online course with the support of virtual resources does not guarantee success. One of the key factors is the motivation of the student. This article provides a brief description of the student's quality perception of the following courses: "Investigacion Educativa" and "Administracion del Curriculo" in Universidad Estatal a Distancia (UNED) in Costa Rica during the academic years of 2012 and 2013; focusing on student's motivation, which is necessary to optimize this virtual learning environment.

Keywords: Education, virtual education, motivation, internet, online course, UNED, Quality.

a un lugar, la disponibilidad de recursos en línea como complemento a los contenidos curriculares, así como la concepción y la mediación constante del docente, son elementos que potencian el aumento en la matrícula de esta modalidad.

Aunado a la anterior, se presenta un cambio en la visión del modelo de formación centrado en la enseñanza 
hacia modelos centrados en el aprendizaje, los estudiantes adquieren roles más participativos y el profesor tutor cada vez más se convierte en un verdadero facilitador de los conocimientos. Por tanto, la motivación que brinde el profesional en educación hacia el aprendizaje de los estudiantes es crucial dentro de la plataforma, pues desde la interacción constante que se construye entre tutor - estudiante - contenidos - plataforma en línea, el aprendizaje significativo se va gestando.

El presente escrito versa sobre tres áreas desarrolladas de forma conexa: una muy breve contextualización sobre las asignaturas en línea de la Cátedra de Investigación Educativa de la Escuela de Educación (ECE) de la Universidad Estatal a Distancia, un análisis de los referentes bases de la motivación y la calidad en los entornos en línea; por último, algunas experiencias de los participantes de diferentes procesos formativos que ratifican la importancia de la motivación como elemento clave para la calidad en un curso virtual.

Se desarrolla una metodología basada en el estudio descriptivo de fuentes primarias y secundarias de las asignaturas de "Investigación Educativa" y "Administración del currículo" durante los años académicos 2012 y 2013, es decir, las percepciones de algunos participantes de las asignaturas, así como literatura referida a la temáticas de otros autores. El objetivo planteado fue analizar la percepción de un curso de calidad con base en los procesos formativos "Investigación Educativa" y "Administración del currículo" de la UNED, de acuerdo con la motivación de los estudiantes de las asignaturas señaladas.

\section{MARCO TEÓRICO}

\section{Calidad de la educación}

El uso intensivo que se ha mantenido en los últimos años sobre el concepto de calidad continúa siendo tema de discusión. Parafraseando a Bensimon (1995), la calidad, al igual que otros aportes a la educación, se refiere a un significado no acabado, ciertamente inestable y que se ajusta según la visión que tenga la institución o persona que lo trabaje. La Organización para la Cooperación y el Desarrollo Económico (OCDE, 1995) define la calidad en la educación como aquella que "asegura a todos los jóvenes la adquisición de los conocimientos, capacidades destrezas y actitudes necesarias para equipararles para la vida adulta" (p. 96).

En 1996, el Informe Delors subraya que la educación tiene cuatro grandes pilares o aprendizajes: aprender a conocer, aprender a hacer, aprender a convivir con los demás y aprender a ser. Por lo tanto, la calidad en la educación no resulta sólo del éxito en una de estas áreas. Por último, el concepto de calidad en la educación propuesto por la UNESCO (2005) es multidimensional, es decir, hay cinco factores que influyen en la calidad de la educación:

- Características de los educandos

- Aportes materiales y humanos

- Enseñanza y aprendizaje

- Resultados

- Contexto

Al valorar las posiciones y contrastarlas con la propuesta teórica que hace Josep Duart para el abordaje de la motivación (presentada más adelante), se puede inferir que existe cierta complementariedad entre motivación y calidad pues se coincide en que las percepciones no siempre son las mismas pues dependen de una serie de factores tanto internos como externos propios del sujeto, la acción, el proyecto, el programa, el centro o la asignatura que se evalúe.

Al respecto, el escrito que se presenta no se vincula con ninguna posición acreditadora de la calidad, sino lo que interesa es conocer las percepciones acerca de lo que es calidad de un curso en línea para un grupo de estudiantes universitarios; que si bien es cierto no es un único criterio ni el definitivo, sí es un aporte para conocer desde la perspectiva de ese "otro" si existe o no una percepción de calidad. Es decir, se presenta una noción de calidad de la educación desde un enfoque donde los componentes para su estudio tienen que ver con el contexto (conocimiento del entorno, en este caso el virtual), pero también desde el ser accesible a todas las personas y desde una perspectiva de uso y amigabilidad (que a la postre genera afinidad y motivación).

\section{Gestión de la calidad en los entornos virtuales}

Quienes se dedican a estudiar la calidad, particularmente en los entornos virtuales, señalan que por las características propias de la modalidad no se le puede 
aplicar la misma reflexión que a los estudios de calidad en educación de manera presencial. En muchos lugares la preocupación por cómo evaluar, asegurar y acreditar esa calidad ha sido objeto de estudio e incluso de propuestas específicas. El objetivo de estos modelos es implementar mecanismos de evaluación y control de la calidad de la educación superior con componente virtual, de modo que haya un registro constante del desempeño de esas instituciones y ofertas formativas. Sangrà (2002) señala que

existen dos tendencias básicas en cuanto a la relación entre la determinación de la calidad de la educación virtual: "quienes la consideran un instrumento auxiliar de la presencialidad y quienes la conciben como una entidad con especificidad propia. No obstante, la tendencia predominante que se afirmará cada vez con mayor fuerza es la segunda". Otros autores coinciden también en la especificidad de la educación virtual, sea esta presencial o a distancia (Middlehurst, 2001; Duart y Martínez, 2002), Barbera et al., 2002; Hope, 2001 y PREAU, 2002) (p. 56).

Como se observa, existen diferentes apreciaciones sobre como visualizar la calidad en los entornos virtuales, por ejemplo, parafraseando a Sangrà (2002) la educación por entornos virtuales debe evaluarse de acuerdo con criterios en cuanto a la oferta formativa, y su pertinencia en relación con necesidades sociales y de mercado laboral, la organización y tecnología, los materiales, la docencia y la creación de conocimiento. Precisamente, esa propuesta es la que se acoge parcialmente en esta investigación, pero uniéndola con los postulados de Duart sobre motivación, de modo que no es interés de este estudio hacer una verificación o corroboración con criterios de calidad propuestos por agencias o modelos, sino generar un insumo para concebir la percepción de calidad por parte de los estudiantes sobre una asignatura híbrida e introducirlo al debate actual sobre calidad de la educación.

En el sentido anterior, parafraseando también a Barberà, Garganté y Mominó (2002), para determinar la calidad de la educación en entornos virtuales se necesitan criterios y modelos de calidad derivados de la psicología de la educación, basados en la evaluación de la dinámica de las principales formas de interacción en un ambiente virtual, a saber, las interacciones dinámicas e interdependientes: entre materiales y estudiantes-profesor, entre estudiantes y profesor y entre los propios estudiantes; todo desde la misma motivación que tiene el estudiante hacia esos elemento.

Silvio (2005), citando a Hope (2001) señala que

todos los productos de aprendizaje son una combinación o sistema de insumos, recursos, procesos y prácticas. Si bien todos son significativos, desde el punto de vista del estudiante, como usuario y consumidor principal, los resultados son lo más importante, luego los procesos y las prácticas y finalmente los insumos y recursos que se utilizan en el diseño, la producción y la entrega del producto o servicio de aprendizaje (p. 89).

\section{La motivación: un elemento clave para la percepción de la calidad en una asignatura en línea}

La motivación ha sido tratada desde diferentes perspectivas por cada una de las teorías de aprendizaje más importantes, por ejemplo, Araujo y Chadwick (1988) señalan que para Ausubel la motivación es tanto causa como efecto del aprendizaje, pero para Bruner el mismo aprendizaje es motivación. Parafraseando a Duart (2000) es importante indicar que quien aprende lo hace porque tiene un estímulo para hacerlo, es decir, significa el objeto de estudio porque existe un elemento adicional para realizar tal acción, bien sea para mejorar profesionalmente o en el ámbito laboral; sin embargo, detrás de esa perspectiva siempre existe la motivación innata del ser humano por aprender.

Desde esta perspectiva, deben conjugarse una serie de factores para mantener esa motivación en los estudiantes que abarca la propuesta formativa, los recursos didácticos, la plataforma de aprendizaje, los recursos tecnológicos adicionales y la mediación del profesor tutor, entre otros. Incluso, cita Duart (2000) "internet ahora, como herramienta para la formación, es un elemento de motivación añadido que se debe tener presente, pero que hay que situar en su lugar, y éste es complementario o facilitador del proceso de aprendizaje" (p. 88).

Con base en esta breve motivación relacionada con el aprendizaje, habría que preguntarse, ¿qué es motivación? De acuerdo con la Real Academia Española de 
la Lengua (2013) la motivación es "un ensayo mental preparatorio de una acción para animarse a ejecutarla con interés y diligencia". Ahora bien, es necesario agregar que para motivarse también se requiere querer estarlo y que la misma no es una condición estable, sino dinámica, puede cambiar según las circunstancias que le rodean. Bajo esta condición es importante entonces considerar que se debe:

- Querer estar motivado (aproximarse a la motivación).

- Tener una acción interna y externa, es decir, querer motivarse por sí mismo para lograr algo, pero también condiciones que inciten a seguir adelante con el proceso.

- Establecer una meta que alcanzar para poder seguir por el camino que se desee planificar.

Desde esta perspectiva, la motivación también puede verse como un reto que los estudiantes se pueden plantear en las asignaturas en línea, situación que se ha visto en las percepciones de los estudiantes de la Cátedra de Investigación Educativa de la UNED, potenciando desde su perspectiva la zona de desarrollo próximo (ZDP) el aprendizaje. Vygotsky, citado por Bryndum (2005), menciona:

La zona de desarrollo próximo define aquellas funciones que aún no han madurado, pero se hallan en proceso de maduración; funciones que han de madurar mañana, pero que ahora se encuentran sólo en estado embrionario. Esas funciones podrían ser descritas como los "brotes" o las "flores" del desarrollo, más bien que como los "frutos" del desarrollo. (p. 5)

Esta cita del autor es importante desde la perspectiva motivacional porque si existe un constante acompañamiento en el curso, materiales accesibles y dinámicos y entornos usables, el interés por aprender se mantiene por causas internas y externas, por tal razón al finalizar el mismo arrojará una sensación - producto positivo y de calidad en quien aprende.

Por otro lado, siguiendo la propuesta de Duart (2000, p. 88), es importante comprender la motivación como elemento potenciador de la calidad en un entorno en línea, desde tres perspectivas por detallar:
- La motivación que reside en quien aprende (el estudiante).

- $\quad$ La que reside en el material formativo (material didáctico, plan de trabajo, guía de aprendizaje).

- La que reside y actúa intercediendo la acción docente (la tarea del profesor o formador).

A partir de lo anterior, como ya se explicó, si bien es cierto, la percepción de calidad a partir de la motivación de los estudiantes no es un criterio para indicar si un entorno en línea cumple con parámetros establecidos por agencias certificadoras, sí es un referente por considerar. Por ello, en el caso del estudiante universitario, señalan Rinualdo et al. (2003):

Parece haber coincidencia entre los distintos autores en vincular a la motivación intrínseca con aquellas acciones realizadas por el interés que genera la propia actividad, considerada como un fin en sí misma y no como un medio para alcanzar otras metas. En cambio, la orientación motivacional extrínseca, se caracteriza generalmente como aquella que lleva al individuo a realizar una determinada acción para satisfacer otros motivos que no están relacionados con la actividad en sí misma, sino más bien con la consecución de otras metas que en el campo escolar suelen fijarse en obtener buenas notas, lograr reconocimiento de su educación, ganar recompensas, etcétera. (p. 108)

Es entonces en la motivación extrínseca donde radica la importancia de conocer y valorar la motivación del estudiante para poder evidenciar su percepción de calidad, pues él lo que busca es un reconocimiento de su formación, aspecto que tiene su fundamento en cómo se desarrolla la asignatura.

\section{ASPECTOS METODOLÓGICOS}

La investigación que sustenta esta producción académica se basa en un enfoque cuantitativo de tipo descriptivo pues interesó describir las situaciones tal cual fueron sucediendo en los contextos educativos desarrollados. En ese sentido, mediante la técnica de encuesta se aplicaron cuestionarios para poder conocer la percepción del estudiante acerca de la calidad de una asignatura con base en su motivación al finalizar el 
proceso, pero también, mediante el análisis de contenido cuantitativo y una matriz que se diseño para el efecto se cotejaron las respuestas entre las asignaturas y lo propuesto por Duart (2000). Las variables estudiadas fueron: percepción de calidad del estudiante, relación entre percepción de calidad y la motivación brindada por los recursos educativos, y la relación entre percepción de calidad y la motivación brindada por la mediación pedagógica.

En relación con la población del estudio fueron los estudiantes matriculados en las asignaturas "Administración del currículo" e "Investigación Educativa" durante el tercer cuatrimestre 2012 y el primer cuatrimestre 2013, que en su total sumaron cuatrocientos veintiocho estudiantes, considerando una muestra probabilística de setenta y dos estudiantes y de $95 \%$ de nivel de confianza, para un intervalo de confianza de $10 \%$.

Por último, en relación con los instrumentos se aplicó un cuestionario para conocer las percepciones señaladas en las variables. Al respecto, Barrantes (2010) afirma que la función básica del cuestionario es obtener, por medio de la formulación de preguntas adecuadas, las respuestas que suministren los datos necesarios, para cumplir con lo que se pretende de la investigación. De manera adicional, se aplicó una lista de cotejo para lograr evidencias propiamente de la plataforma de aprendizaje en línea y contrastarlo con lo anotado por los estudiantes en el cuestionario. Asimismo, una matriz de análisis de contenidos cuantitativo para la revisión de respuestas de estudiantes y su relación con la teoría.

\section{RESULTADOS}

Esta sección presenta los principales resultados de los instrumentos aplicados, al respecto, en términos generales se evidencia que existe una adecuada percepción de calidad en los cursos "Investigación Educativa" y "Administración del currículo" de la UNED, de acuerdo con la motivación que expresan los estudiantes de las asignaturas señaladas. Al respecto, de acuerdo con Ruiz (2011):

La educación superior del siglo XXI (...) debe promover aquellos saberes que proponía Jacques Delors (1996): el aprender a hacer, aprender a ser, aprender a conocer y aprender a convivir. En este sentido, la Universidad Estatal a Distancia (UNED) de Costa Rica ha dirigido esfuerzos para presentar en las políticas institucionales y en su Modelo Pedagógico este paradigma (centrado en quien aprende), en el cual los estudiantes no solo tratan de desarrollar esos saberes, sino que los profesionales que desempeñan cargos docentes se desenvuelven y se actualizan en torno a esta dirección." (p. 2)

Durante el año 2012 y 2013 varias de las asignaturas se ofertan bajo la modalidad virtual, es decir, un cien por ciento de las actividades se realizan en un entorno de aprendizaje, en este caso, la plataforma Moodle de la Universidad. Para el caso que compete las asignaturas "Investigación Educativa" y "Administración del currícuIo" tanto la matriculación, como las consultas, actividades y entrega de tareas y resultados, son desarrolladas completamente en línea ingresando en la página web de la Universidad (www.uned.ac.cr) y luego a la sección "Recursos Didácticos" (http://recdidacticos.uned.ac.cr/ $\mathrm{pal} /$ ). En términos generales, estas asignaturas fueron diseñadas de modo que las actividades de aprendizaje potencien la construcción individual y, en algunos casos, colaborativa del conocimiento. En el cuadro 1 se detallan las actividades.

En términos generales las actividades promueven habilidades investigativas por parte del estudiante pero desde su cotidianidad, pues en unos casos contextualiza el currículo oficial del Ministerio de Educación Pública (MEP) para solventar problemas de pertinencia cultural mientras en otros desarrolla diseños de investigación para comprender mejor situaciones cotidianas en sus aulas; razones por las cuales se concibe la investigación como un proceso clave no solo para el ejercicio profesional, sino también para mejorar sus experiencias de vida, aspectos que según los mismos estudiantes les motivan a continuar estudiando, desarrollar prácticas exitosas y considerar los asignaturas como positivas o de calidad. (Cuadro 2)

Evidentemente, por los comentarios de los participantes, existe una motivación por parte del alumnado hacia las actividades desarrolladas y hacia la mediación de los profesores tutores de los asignaturas, siendo esto, entonces, un indicador que permite evidenciar asignaturas de calidad pues facilita la tarea de construir el aprendizaje, tanto de manera individual como colaborativa. Asimismo, citando a Barrientos y Carillo (2011): 
CUADRO 1

Actividades de las asignaturas "Investigación Educativa" y "Administración del currículo" durante los años académicos 2012 y 2013. Cátedra de Investigación Educativa, UNED

\begin{tabular}{cll}
\multicolumn{1}{c}{ Asignatura } & \multicolumn{1}{c}{$\begin{array}{c}\text { Actividades didácticas realizadas } \\
\text { por los estudiantes en el entorno virtual }\end{array}$} & Período \\
Administración del currículo (325) & $\begin{array}{l}\text { Trabajo de análisis teórico } \\
\text { Proyecto de contextualización curricular }\end{array}$ & $\begin{array}{l}\text { Tercer cuatrimestre } \\
2012 \text { y primer } \\
\text { cuatrimestre 2013 }\end{array}$ \\
& $\begin{array}{l}\text { Foros de discusión: académicos, sociales y de dudas } \\
\text { Ensayo: construcción colaborativa }\end{array}$ & Tercer cuatrimestre \\
& Tarea: reflexión de investigación & 2012 y primer \\
& Revisión de literatura: búsqueda colaborativa de & cuatrimestre 2013 \\
& información documental & \\
& Diseño de un proyecto de investigación & \\
& Foros de discusión: académicos, sociales y de dudas & \\
\hline
\end{tabular}

Fuente: Elaboración propia con base en las orientaciones académicas de los asignaturas durante el año académico 2012 y 2013.

La confianza y la atención, son componentes que contribuyen al interés y esfuerzo que un estudiante realiza en un curso en línea. La motivación proporciona confianza, seguridad, apoyo, deseo de continuar estudiando, etcétera. Este elemento juega un papel importante en la enseñanza, ya que la meta no solo es ayudar y asesorar para que los alumnos terminen el curso, sino también para obtener resultados reales y duraderos. (p. 33)

Es por lo anterior que la motivación también influye para obtener indicadores de calidad para juzgar, evaluar y percibir un curso en línea.

\section{La motivación como una percepción de calidad en las asignaturas en línea de "Investigación Educativa" y "Administración del Currículo"}

\section{a) La motivación del estudiante}

De acuerdo con Duart (2000) la motivación del estudiante para evidenciar percepciones de calidad en una oferta formativa se pueden resumir en tres aspectos: "la necesidad de formación, el interés por el contenido y la significación del aprendizaje (p. 89)". Bajo esta premisa, los estudiantes de las asignaturas mencionadas de la Cátedra de Investigación Educativa señalan que bajo esta modalidad las mismas asignaturas son muy buenas debido a los razonamientos expuestos en el cuadro 3.

Obsérvese que los comentarios de los estudiantes giran en torno a situaciones que le representaron significatividad y por ende motivación para finalizar con éxito las asignaturas en línea:

- Imposibilidad de asistir físicamente a un centro de estudio.

- Necesidad de obtener un resultado con cierta celeridad.

- Ampliación del ámbito formativo a otros procesos formativos de la carrera.

- Utilización de elementos del curso en su vida personal (por ejemplo, mejorar vocabulario).

- Autorregulación de las responsabilidades.

Existe, entonces, una necesidad por parte de los estudiantes que manifiestan su deseo de aprender y saben sobre la utilidad de los conocimientos que van adquiriendo, hecho sin duda motivador para el proceso y por lo tanto indicador de calidad desde su perspectiva. Se evidencia la motivación a raíz de una identificación de lo aprendido, pero también en relación con aplicaciones futuras.

\section{b) La motivación desde la metodología y los materiales del curso}




\section{CUADRO 2}

Percepciones de los estudiantes referidos a la calidad del curso. Tercer trimestre 2012 y primer cuatrimestre 2013

\begin{tabular}{|c|c|}
\hline Asignaturas & $\begin{array}{l}\text { Percepciones de los estudiantes } \\
\text { Tercer trimestre } 2012 \text { y primer cuatrimestre } 2013\end{array}$ \\
\hline \multirow[t]{4}{*}{ Administracion del círculo } & $\begin{array}{l}\text { Estudiante 1. } \\
\text { "La facilidad (...) es que todo se realiza por medio de la web pero igual la profundidad del mismo } \\
\text { es su dificultad, pero en general la experiencia y el aprendizaje fue de mucho agrado por que nos } \\
\text { actualiza principalmente en el tema del currículo, el cual es punto importante en mi formación } \\
\text { como docente ya que esos cambios se formulan para cubrir las necesidades de los estudiantes ya } \\
\text { sean regulares o con necesidades educativas especiales, tomando en cuenta la participación de } \\
\text { los padres de familia, es un curso bastante útil". }\end{array}$ \\
\hline & $\begin{array}{l}\text { Estudiante } 2 . \\
\text { "La metodología utilizada por el curso es muy buena, le permite a uno como docente o futuro } \\
\text { docente obtener más conocimientos, ya que uno tiene que leer información muy valiosa, in- } \\
\text { vestigar y compartir opiniones con los demás compañeros y el profesor, además de un mayor } \\
\text { acercamiento con los recursos tecnológicos". }\end{array}$ \\
\hline & $\begin{array}{l}\text { Estudiante } 3 . \\
\text { "En mi opinión el curso es excelente y solo sugiero que sigan siendo responsables con la atención } \\
\text { a las dudas, revisión de trabajos y de foros". }\end{array}$ \\
\hline & $\begin{array}{l}\text { Estudiante } 4 \text {. } \\
\text { "El curso me parece muy bueno y provechoso, de esta modalidad virtual puede uno extraer ma- } \\
\text { yores contenidos, abarcarlos e interiorizarlos adecuadamente, la metodología en la que se de- } \\
\text { sarrolló me parece la adecuada, en forma de lectura de diferentes textos y siempre los tutores } \\
\text { anuentes a aclarar duras, muy amables y profesionales, debo agradecerles mucho la interacción } \\
\text { con los compañeros, que todos con sus comentarios respetuosos interactuaban, así como moti- } \\
\text { vaban para seguir adelante, las actividades fueron adecuadas a los temas, la programación fue } \\
\text { muy bien planeada y diseñada". }\end{array}$ \\
\hline
\end{tabular}

Investigación educativa

\section{Estudiante 1.}

“Considero que una de las mayores fortalezas es la metodología que se emplea en el desarrollo de las diversas actividades, porque permite la interacción entre todos los participantes, estudiantes y profesor. Esto favorece en el proceso de aprendizaje porque permite que todos de una manera dinámica nos retroalimentemos de los diferentes conocimientos y puntos de vista que cada uno tenemos con respecto a un determinado asunto. Otro aspecto es que hacemos uso de los recursos tecnológicos, como en este caso la Internet, es una forma importante de innovar la educación y salirnos del tradicional estilo de aprender".

\section{Estudiante 2.}

"Creo que el curso se desarrolló de una manera excelente, sus actividades fueron de mucho aprendizaje, el compartir con mis compañeros me dio mucho más de lo que esperaba en lo que conocimiento se refiere, creo que si hubiera alguna sugerencia sería que se tenga todavía más contacto con el tutor".

\section{Estudiante 3.}

"Quiero manifestar mi satisfacción por haber realizado y casi finalizado este curso, realmente aprendí bastante, logré finalmente comprender como funciona la biblioteca virtual y la revisión de literatura".

\section{Estudiante 4.}

"Hola, profesor, espero que se encuentre bien, deseando éxitos en sus labore, la presente es para comunicarle que el curso me gustó mucho, porque nunca había participado de este modo, como fortaleza me gustaron todas las actividades para la realización de este, por lo cual estoy muy agradecida".

Fuente: Elaboración propia con base en el foro de evaluación del curso disponible en todos los asignaturas en línea de la Cátedra al finalizar cada cuatrimestre. 


\section{CUADRO 3}

Percepciones de los estudiantes con respecto a su motivación y qué les llevó a tener una percepción de calidad de la asignatura. Primer cuatrimestre 2013

Asignaturas

Percepciones de los estudiantes. Primer cuatrimestre 2013

Administración del Currículo Estudiante 1.

e

“Bueno hemos llegado al cierre de un proceso, muy enriquecedor, puedo decir que me sirvió de-

Investigación Educativa masiado en el campo profesional. Además como ser humano me dio la oportunidad de conocer personas responsables con su trabajo, tanto los compañeros como la profesora. En asignaturas anteriores no he querido hacer la evaluación del curso debido al malestar con la plataforma y la profesora pero en esta oportunidad he quedado muy contento, es un curso muy bueno".

\section{Estudiante 2.}

“En lo que respecta a contenidos, metodología, actividades, programación desde el punto de vista personal considero que se han planteado de una manera apropiada porque le permite a una organizarse mejor con el tiempo, no solo de estudio sino con las otras obligaciones personal y laborales".

\section{Estudiante 3.}

“Una fortaleza es la posibilidad de llevar el curso de manera virtual ya que a pesar de las facilidades que nos brinda la UNED, algunas personas, como me sucede a mí, no podemos asistir a las tutorías por cuestiones laborales, siendo importante que hagan más asignaturas de este tipo, por lo demás excelente curso".

\section{Estudiante 4.}

"Al principio del curso tenía mucho miedo porque no sabía de qué se trataba y poco a poco me fui adaptando al sistema y sé que no me fue muy bien en algunas tareas; eso me llevo a reflexionar sobre mis errores y cómo hacer mejor las cosas con mi estudio; me gustaría darle las gracias a la tutora porque se ha comportado muy paciente conmigo en los foros y las tareas; cursos como este hacen que uno se emocione más".

\section{Estudiante 5.}

"A mi parecer este curso se facilitó al ser virtual ya que fue más fácil realizar preguntas y las dudas que tenía me las aclaraban rápidamente".

\section{Estudiante 6.}

"Ha sido un curso excelente, donde he aprendido a planear, organizar, estructurar y aplicar mi estudio. En mi caso, me he encontrado con algunas dificultades, primero que salí del colegio hace treinta años, me ha costado acoplarme, luego soy una persona muy sencilla o sea que escribo a como hablo y en el transcurso del cuatrimestre aprendí que aparte de aprender a desarrollar un proceso de investigación, que debía mejorar y ampliar mi vocabulario. Así que he aprendido algunas cosas extras, definitivamente me quedo con la calidad de la UNED".

Fuente: Elaboración propia con base en el foro de evaluación del curso disponible en todas las asignaturas en línea de la Cátedra al finalizar cada cuatrimestre.

Los recursos didácticos presentes en los entornos en línea siempre deben tener de manera explícita elementos que motiven su utilización y por tanto estimulen el aprendizaje. En la Cátedra de Investigación Educativa de la UNED se persigue que los materiales sean lo suficientemente atractivos para que no solo sean provechosos en las actividades programadas, sino para la vida profesional y personal del estudiante; en este caso, se considera que se han relacionado estas dos situaciones de manera acertada y óptima.
Algunas características del material utilizado son:

- Estar diseñado para estudiantes que no cuentan con una figura docente a tiempo completo para aclarar sus dudas.

- Ser comprendido en poco tiempo de acuerdo con el perfil general del estudiante.

- Ser claro, conciso y puntual sobre el objetivo de aprendizaje propuesto. 
- $\quad$ Ser accesible en el momento en que se requiera.

- Estar organizado por objetivos, contenidos, ejemplos, imágenes, ejercicios de autoevaluación, entre otros.

En el anterior sentido, los estudiantes demuestran lo señalado, nótese su percepción en el cuadro 4.

Con base en las observaciones de los estudiantes, se puede deducir que los materiales utilizados en las asignaturas "Administración del currículo" e "Investigación
Educativa" han sido diseñados pensando en la modalidad educativa que cursan: la educación a distancia y en este caso con apoyo de recursos tecnológicos. Esto a pesar de que no todos fueron producidos por la Universidad; por ejemplo, en el curso "Administración del Currículo" se utilizan lecturas de autores ajenos a la UNED. Solamente en el curso "Investigación Educativa" se emplea una unidad didáctica modular producida por la Dirección de Producción de Material Didáctico (DPMD) de la UNED. De acuerdo con Duart (2000):

\section{CUADRO 4}

Percepciones de los estudiantes con respecto a su motivación en función de los materiales didácticos utilizados. Primer cuatrimestre 2013

\section{Asignaturas}

\section{Percepciones de los estudiantes. Primer cuatrimestre 2013}

\section{Administración del Currículo Estudiante 1.}

e "El curso en sí fue muy interesante porque aprendí muchas cosas, las actividades muy buenas de Investigación Educativa acuerdo con los contenidos de los materiales; pusimos en práctica los conocimientos adquiridos. Espero que pueda desarrollar lo aprendido cuando esté trabajando que es donde nos damos cuenta cuánto sabemos y hemos aprendido".

\section{Estudiante 2.}

"Los materiales utilizados en el curso le permite a uno como docente o futuro docente obtener más conocimientos, ya que uno tiene que leer información muy valiosa, investigar y compartir opiniones con los demás compañeros y el profesor, además de un mayor acercamiento con los recursos tecnológicos".

\section{Estudiante 3.}

"La manera tan interactiva del curso me parece muy importante, otra fortaleza es la retroalimentación que se hace por medio de los foros y la participación de los compañeros".

\section{Estudiante 4.}

“En la metodología, el desarrollo de productos creativos provoca interés en la investigación y esmero en la presentación del trabajo. La programación se cumplió a cabalidad según los recursos que nos dieron".

\section{Estudiante 5.}

"El presente curso trajo consigo muchos aprendizajes y muchos retos entre los cuales a mi parecer sus principales fortalezas son la plataforma, que permite tener un buen contacto con el tutor encargado, la metodología que es algo compleja, pero los contenidos se ajustan a las diferentes actividades".

\section{Estudiante 6.}

"Las actividades estuvieron muy acordes con los contenidos de la unidad, además de promover siempre la investigación de acuerdo con el libro del curso".

\section{Estudiante 7.}

“ßBuenas! El curso en línea, Investigación Educativa, fue mi primera experiencia, considero que es una manera más fácil y accesible para estudiar a distancia, no demanda mucho tiempo lo que favorece a los que se encuentran laborando, las actividades y programación están bien distribuidas, además me agrada que el estudiante pueda ir conociendo su calificación. Los materiales fueron sencillos y claros".

Fuente: Elaboración propia con base en el foro de evaluación del curso disponible en todos los asignaturas en línea de la Cátedra al finalizar cada cuatrimestre. 
Un buen y motivador diseño formativo de un material didáctico para la formación no presencial no es el que incluye mucho y diversificados elementos multimedia, sino el que los incluye en la medida necesaria, es decir, de acuerdo con la tipología de contenidos, los objetivos de aprendizaje y las finalidades formativas concretas. (pág. 91)

Los siguientes son algunos aspectos que resaltan de las percepciones de los estudiantes con respecto a los materiales y que evidencian su motivación:

- Facilidad de acceso

- Utilización clara de los recursos

- Navegabilidad sencilla
- Exposición metódica y concreta del recurso

- Promoción del autoaprendizaje

En suma, hay una aprobación de los materiales por parte de los estudiantes, los encuentran relevantes para las actividades propuestas y en general para su vida profesional y personal, siendo de esta manera un elemento motivador más que provoca una satisfacción por parte de quien aprende, elemento necesario para el aseguramiento de la calidad.

\section{c) La motivación por parte del profesor tutor}

La mediación docente en el curso virtual es clave para fomentar la motivación externa del estudiante,

CUADRO 5

Percepciones de los estudiantes con respecto a su motivación en función de la mediación pedagógica. Año académico 2013.

Asignaturas Administración del Currículo e Investigación Educativa
Percepciones de los estudiantes. Año académico 2013.

Estudiante 1.

El curso me parece muy provechoso, de esta modalidad virtual puede uno extraer mayores contenidos, abarcarlos e interiorizarlos adecuadamente, la metodología en la que se desarrolló me parece la adecuada, en forma de lectura de diferentes textos y siempre los tutores anuentes a aclarar dudas, muy amables y profesionales, debo agradecerles mucho, la interacción con los compañeros, que todos con sus comentarios respetuosos interactuaban, así como motivaban para seguir adelante, las actividades fueron adecuadas a los temas, la programación fue muy bien planeada y diseñada.

\section{Estudiante 2.}

Buenas, profesor, me encuentro muy satisfecha con este curso, ya que permite en gran medida aprender sobre los trabajos de investigación, por otro lado me siento muy agradecida con usted ya que siempre estuvo dispuesto a colaborar con cualquier duda, lo que lo ayuda a uno como estudiantes, sentir que cuenta con alguien que lo pueda guiar.

\section{Estudiante 3.}

Hola:

Apoyo y secundo tu comentario, en realidad la plataforma está muy completa y clara y el trabajo del profesaor (sic) a sido estupendo, ojalá y se pueda reconocer el trabajo y la entrega del profe ya que gracias a él nosotras como estudiantes estamos motivadas.

\section{Estudiante 4.}

Considero que fue un curso muy provechoso, el profesor siempre estuvo dando seguimiento a todo el proceso, atendiendo dudas y retro alimentándonos en forma constante. Por eso mi agradecimiento para el profesor por todo su apoyo y su excelente trabajo.

\section{Estudiante 5.}

Quiero agradecer al profesor, por el apoyo a lo largo de este curso, excelente trabajo como guía y orientador en el proceso de enseñanza - aprendizaje.

Fuente: Elaboración propia con base en el foro de evaluación del curso disponible en todos los asignaturas en línea de la Cátedra al finalizar cada cuatrimestre. 
pues no solo es aclarar dudas y orientar, sino brindar ese estímulo extra para impulsar la motivación innata del estudiante.

La orientación, la guía, el estímulo y la motivación del profesor tutor, menciona Duart (2000), son claves en las asignaturas virtuales para generar una percepción de calidad, dado que la relación entre el profesor y el estudiante se produce de manera asincrónica.

En relación con lo anterior, es importante aclarar que para las asignaturas estudiadas la mediación pedagógica fue desarrollada en su totalidad en el entorno virtual en línea para atender las actividades señaladas en el cuadro 1. A partir de estas actividades, la persona tutora pudo estimular la motivación externa y coadyuvar a generar la percepción de calidad, sujeto de estudio en este trabajo.

Ahora bien, en el cuadro 5 se evidencian algunas percepciones finales de los estudiantes con respecto a su profesor tutor, una vez realizada la evaluación del curso.

Nótese como las percepciones de los estudiantes señalan la importancia de la mediación pedagógica en el impulso de los tres autos del aprendizaje en educación a distancia: autorregulación, autonomía y autoaprendizaje. La motivación pues radica entonces en hacer sentir cómodo al estudiante, pero desde contextos de aprendizaje; es decir, no se trata de captar la atención o el interés del estudiante, sino de la capacidad de orientarlo hacia el desarrollo de habilidades para su mejora académica.

\section{CONCLUSIONES}

Las relaciones antes anotadas: motivación personal (intrínseco), recursos educativos (extrínseco) y mediación pedagógica del profesor tutor (extrínseco) son elementos que se determinan a partir de la motivación de los estudiantes en las asignaturas "Administración del Currículo" e "Investigación Educativa" que impartió la UNED durante los años 2012 y 2013, mediante una percepción de calidad en las mismas.

La motivación como eje central no solo se halla en un elemento, sino que se evidencia en todos los componentes del aprendizaje y es a partir de la interacción de todos los actores del proceso formativo: tutor - estudiante - contenidos - plataforma en línea que se gestan aprendizajes individuales y colectivos de manera significativa. Como señala Duart (2000):

El uso de simulaciones, el trabajo en equipo y la interactividad, el uso de hipermedia, y el uso de recursos gráficos o audiovisuales que estén a nuestro alcance con la finalidad de llamar la atención y de hacer más significativo el proceso de aprendizaje, sin duda ayudan a mantener la motivación hacia el mismo. (p. 107)

Al relacionar lo anotado por Duart con la definición de calidad educativa de la UNESCO, se evidencia un carácter multidimensional que gesta percepciones de calidad a partir de las características de los educandos, los aportes materiales y humanos, la forma de enseñanza y el aprendizaje, los resultados y por supuesto el contexto en donde se desarrolla.

Ahora bien, aunque la percepción de calidad no es el único elemento que puede utilizarse para señalar la calidad de un proceso formativo, si es uno de los aspectos que se consideran a nivel internacional en procesos de acreditación, razón por la cual este estudio es un insumo que aporta conocimiento y metodología al proceso, siendo necesario ampliar la población y el objeto de estudio a otras asignaturas, de modo que se logren resultados más amplios y generalizables.

Del mismo modo, se requiere identificar las buenas prácticas docentes desarrolladas en las asignaturas de la Cátedra para poder brindar insumos que complementen la percepción del estudiante, que fue el eje de este trabajo. Dicho de otra manera, es necesario valorar con más estudios los procesos de mediación pedagógica llevados a cabo para el fomento de la motivación extrínseca en los estudiantes, pero ahora desde la percepción docente, de esta forma se identificarán actividades por replicar, herramientas por sugerir, recursos por utilizar, entre otros; esto es entonces un reto investigativo producto del trabajo presentado.

Para finalizar, es necesario un esfuerzo para que en todos los procesos de creación del curso, por ejemplo, las orientaciones del estudiante, el diseño instruccional del entorno virtual, la asignación de los grupos, la matrícula, entre otros, se tome en cuenta la explicitación de generar motivación extrínseca en el estudiante para que la dinámica educativa siempre busque el beneficio del estudiante como eje clave de todo proceso académico inspirado en el Modelo Pedagógico de la Universidad Estatal a Distancia. 


\section{REFERENCIAS}

Araujo, J. y Chadwick, C. (1988). Tecnología educacional. Barcelona: Paidós.

Barrientos, A. y Carrillo, M. (2011). La motivación en las asignaturas en línea. Digital Learning College. Recuperado de http://dlearncollegecom.fatcow.com/blog/?p=184

Bryndum, S. (2005). La motivación en los entornos telemáticos red. Revista de Educación a Distancia. Año V. Número 13. 9 - 24 Recuperado de http://www.um.es/ead/red/13/ bryndum.pdf

Duart, J. (2000). Aprender en la virtualidad. Barcelona: Ed. Gedisa.
Fainholc, B. (1999). La interactividad en la educación a distancia. Buenos Aires: Ed. Paidos.

Rinaudo, M. et al (2003). Motivación y uso de estrategias en estudiantes universitarios. Su evaluación a partir del Motivated Strategies Learning Questionnaire. En Anales de Psicología. Vol. 19. No. 1. Junio. Recuperado de http:// www.um.es/analesps/v19/v19_1/11-19_1.pdf

Ruiz, W. (2011). El Centro de Capacitación en Educación a Distancia: de la capacitación al desarrollo profesional 2000 - 2010. En Revista Educación. 35(1). San José: Universidad de Costa Rica. Recuperado de http://revistas. ucr.ac.cr/index.php/educacion/article/viewFile/464/453 